\title{
Gesundheit als zentrales Thema der Politik - Politik als zentrales Thema der Gesundheit
}

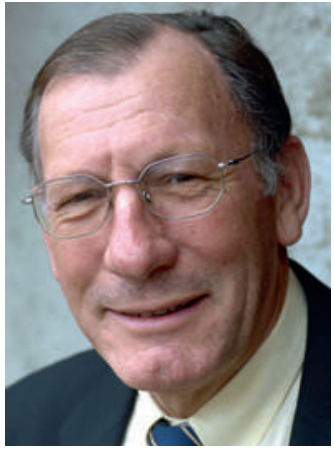

Jean Martin

\section{Literatur}

1 Myser C. (Hrsg). Bioethics around the Globe. New York: Oxford University Press; 2011.

2 Dies bringt uns zur oben beschriebenen juristischen Kontroverse in den USA zurück, die das strikte Primat der Freiheit des Einzelnen bei jedwedem Handeln propagiert, auch inkl. der Freiheit, auf jegliche Absicherung beim Thema Gesundheit zu verzichten, um dann den anderen zur Last zu fallen.

3 Pelluchon C. Eléments pour une éthique de la vulnérabilité.

Paris: Ed. du Cerf; 2011.
Die Gesundheitsversorgung ist ein zentrales Thema der Politik. Im Frühjahr 2012 wird dies besonders offenkundig. Die Verordnungsbestimmung zur Integrierten Versorgung / Managed Care hat in der Schweiz unterschiedliche Optionen, Befürchtungen und Vorgehensweisen innerhalb der Ärzteschaft zutage treten lassen. Der Entwurf für ein Bundesgesetz über die Prävention bewirkte Erstaunliches - darunter $\mathrm{u}$. a. den Nichteintretensentscheid des Ständerats Ende 2011 und die gleichzeitige Wiederaufnahme der Arbeit durch die Kammern. Dabei muss in diesem Kontext wohl nicht daran erinnert werden, wie sehr die verschiedenen Lebensumstände in ihrer sozioökonomischen, kulturellen und physiochemischen Ausprägung (Umweltverschmutzung) die Gesundheit und die Nachfrage nach adäquaten Präventivmassnahmen beeinflussen. Fakt ist jedoch, dass im weitesten Sinne liberale Gesellschaften wie die unsere solchen Massnahmen a priori nicht sehr positiv gegenüberstehen. Sie berufen sich auf die persönliche Freiheit, bewerten sie höher als die erforderlichen «Sicherheitsmechanismen». Dies zulasten späterer Schäden, deren Konsequenzen es dann zu zahlen gilt, ohne dass dabei die menschlichen bzw. sozialen Schäden wirklich repariert werden können. Die FMH unterstützt das Gesetz über die Prävention zu Recht, und man möchte glauben, dass es nach all den Debatten auch endlich angenommen wird.

In den Vereinigten Staaten wird in Bezug auf Einschränkungen der persönlichen Freiheit ähnlich argumentiert, um sich dem Affordable Care Act (Obamacare für die Gesetzesgegner) zu widersetzen. Das Gesetz ist vor zwei Jahren verabschiedet worden. Einige Punkte wurden jedoch vor kurzem in Anhörungen vor dem Obersten Gerichtshof geprüft. Dabei geht es um die Frage, ob das von besagtem Gesetz vorgesehene universal individual mandate verfassungsgemäss ist. Die strittige Bestimmung sieht vor, dass jeder Amerikaner verpflichtet ist, sich auf die eine oder andere Weise gegen Krankheit abzusichern. In der Argumentation dagegen wird häufig die Freiheit, sich ein Auto zu kaufen oder Freizeit in Anspruch zu nehmen, auf dieselbe Argumentationsstufe gestellt wie die Einführung einer für alle geltenden Garantie für eine angemessene Mindestversorgung. Der Zugang zu einer guten Gesundheitsversorgung wird nicht wie in Europa allgemein üblich - als wichtiges soziales Recht angesehen, sondern vielmehr als commodity, die man sich leistet, wenn man die Mittel dazu hat oder auf die man verzichtet, falls diese Mittel nicht vorhanden sind. Der im Grundsatz politische Charakter der Debatte wird durch die Tatsache unter- strichen, dass die in diesem Zusammenhang getroffenen Entscheide erstinstanzlicher Gerichte strikt nach der entsprechenden Wertvorstellung des jeweiligen Richters gefällt wurden. Da im Obersten Gerichtshof fünf Republikaner und vier Demokraten sitzen, besteht die ernsthafte Gefahr, dass das, was als wichtigste US-Sozialgesetzgebung der letzten vierzig Jahre beschrieben wurde, zunichte gemacht wird.

Auf internationaler Ebene überraschte jüngst die Tatsache, dass die USA einen Mediziner als WeltbankPräsidenten vorgeschlagen haben, jener Bank, die zu den wichtigsten mit der Entwicklung armer Länder befassten Organisationen zählt. Gemeint ist Prof. Jim Yong Kim, 53, Amerikaner koreanischer Abstammung, Arzt im öffentlichen Gesundheitswesen und Universitäts-Präsident. Er ist Mitbegründer der Nichtregierungsorganisation Partners in Health, die in vielen Ländern effiziente und wirtschaftliche Programme im Kampf gegen die grossen Infektionskrankheiten eingeführt hat, arbeitete von 2003 bis 2006 für die WHO und lehrte gleichzeitig in Harvard. Bei einer Wahl ist zu hoffen, dass die Institution künftig mehr und kompetenteres Interesse für die Gesundheit als grundlegenden Faktor in der Entwicklung zeigen wird. Möglicherweise erinnert sich Dr. Jim Yong Kim an den Satz von Martin Luther King: «Von allen Formen der Ungleichheit ist Ungerechtigkeit im Gesundheitswesen die unmenschlichste.»

Politik hat immer auch eine ethische Komponente oder sollte sie zumindest haben. Abschliessend möchte ich noch eine Veröffentlichung über die Bioethik in der Welt anführen [1]. Sie untersucht, wie in verschiedenen Kontinenten und Regionen bestimmte ethische Grundprinzipien ungleich gewichtet werden. In Afrika und Asien spielt die Gruppe (Grossfamilie bzw. Gemeinschaft) in der Entscheidungsfindung eine Rolle, die wir ihr in unseren westlich geprägten Vorstellungen und Auffassungen nur schwer zugestehen [2]. Selbstverständlich ist nicht zu billigen, dass ein Mensch auf unzulässige Weise den Entscheidungen anderer unterliegt. Doch Sensibilität für unterschiedliche Bezugsrahmen ist unabdingbar, wenn es darum geht, Modelle zu erarbeiten, die den Bedürfnissen und Lebensumständen aller entsprechen.

In einem anderen, jüngst erschienenen Werk zum Thema Ethik [3] postuliert C. Pelluchon, dass die persönliche Verantwortung des Einzelnen unter den gegebenen weltweiten Bedingungen höher zu werten sei als allein seine Freiheit. Darüber gilt es nachzudenken! und der nationalen Ethikkommission 